\title{
Stress analysis of plate with opposite semicircular notches and adhesively bonded piezoelectric actuators
}

\author{
Ahmed Abuzaid ${ }^{1}$, Meftah Hrairi' ${ }^{2}$ Hashim Kabrein ${ }^{3}$ \\ ${ }^{1,3}$ Safat College of Science and Technology, Khartoum, Sudan \\ ${ }^{2}$ Mechanical Engineering Department, IIUM, P.O. Box 10,50728, Kuala Lumpur, Malaysia \\ ${ }^{1}$ Corresponding author \\ E-mail: ${ }^{1}$ ahmezaid@gmail.com, ${ }^{2}$ meftah@iium.edu.my, ${ }^{3}$ hashimkabrein@gmail.com
}

Received 25 January 2020; accepted 18 February 2020

DOI https://doi.org/10.21595/vp.2020.21311

Check for updates

Copyright (C) 2020 Ahmed Abuzaid, et al. This is an open access article distributed under the Creative Commons Attribution License, which permits unrestricted use, distribution, and reproduction in any medium, provided the original work is properly cited.

\begin{abstract}
Stress control of structures by means of active smart material has evolved in recent years. The structure's stress control can typically be considered as strengthening that effects the structure's load-carrying capability. The stress-concentration cause by holes and notches produces a large variation and significant increase in stress. The control of the stress concentration and its distributions in the notched area is valuable for many practical and design applications. This study investigates the impacts of the piezoelectric actuators on control of the stress concentration factor (SCF) for an aluminum plate with opposite semicircular notches. Finite-element method (FEM) is used to access the SCFs due to external static loading. The stress control efficiency is assessed by the SCFs as an effectiveness criterion. The results indicate that SCFs reduces linearly with active piezoelectric actuator.
\end{abstract}

Keywords: smart material, piezoelectric actuator, stress concentration factor, FEM.

\section{Introduction}

Stress concentration factor is a crucial parameter in the structural analysis, specifically for structural with holes and notches. SCF is the relation between the notch maximum stress and the normal stress when there is no notch [1]. High SCF can lead to initiation and propagation of crack which result in catastrophic failure. Smoothing the stress flow near the geometrical discontinuities can decrease the high SCF. Different methods used to reduce the SCF includes strengthening of the hole by adding composite material [2], using auxiliary holes in the vicinity of the high SCF [3] and optimization of the hole shape [4].

The piezoelectric materials offer distinguished advanced technique to control the high stress issues on the notched structural. This is due to the ability to adjusted the piezoelectric materials to the change in the external load with little growth in the structural weight. Furthermore, in the aerospace and automotive engineering, the structure high stress control using the piezoelectric actuators at the notched position can prolong the service life and cost-effective alternative to restore the structural integrity.

The stress generated by the piezoelectric patches on the host structure can help to mitigate the stress concentration due to the external load. Different methodologies used for the active stress control and repair via the piezoelectric actuator [5-6]. Reduction of the stress concentration near notches and holes using the piezoelectric actuators was investigated by Rogers [7]. The study, conducted by Shah et al. [8], found that $33 \%$ of stress concentration on a finite plate with a hole can be obtain using the active piezoelectric actuator.

Recently, the importance of power, stiffness and placement of the piezoelectric patches on reduction of the SCF around a hole was conducted by Fesharaki et al. [9, 10]. A novel study to reduce SCF constructed on the particle swarm optimization was investigated by Fesharaki and Golabi [11]. Their outcomes indicated that tension stress near a hole can be decrease using specified piezoelectric patches pattern recognition. Abuzaid et al. [12] studied placement of the piezoelectric actuator above a hole in plate subjected to far field tension. Their results showed that, the positive power on the piezoelectric decrease SCF and alliteratively, the negative power on the 
piezoelectric increase SCF. However, most of the previous works are limited to two-dimensional analysis and there is no study on the applications of the piezoelectric actuator stresses on the notch structural.

This work investigates the control of stress concentration using the piezoelectric actuator near the notches that cause a change in the stress distribution and can lead to the crack/damage initiation or propagation in thin plate structures used in spacecraft, aircraft, or automotive industries. For this the aims the piezoelectric effect on the SCF for a plate with opposite semicircular notches integrated with a piezoelectric actuator was studied. The main object is to examine the induce tension/compression stresses by the piezoelectric actuator in the increase/decrease of the SCFs. Finite-element Method (FEM) is used to obtain the SCFs under tension.

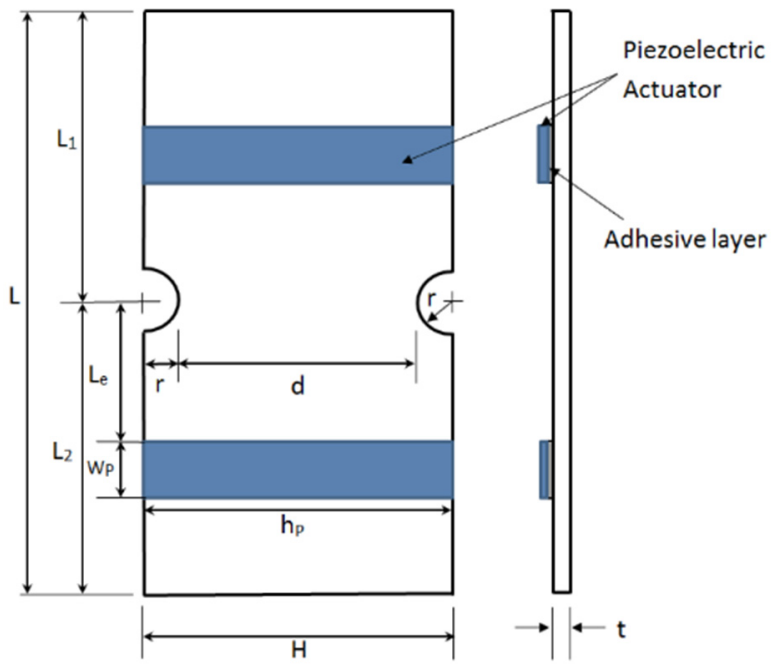

Fig. 1. Plate with opposite semicircular notches and integrated piezoelectric patch

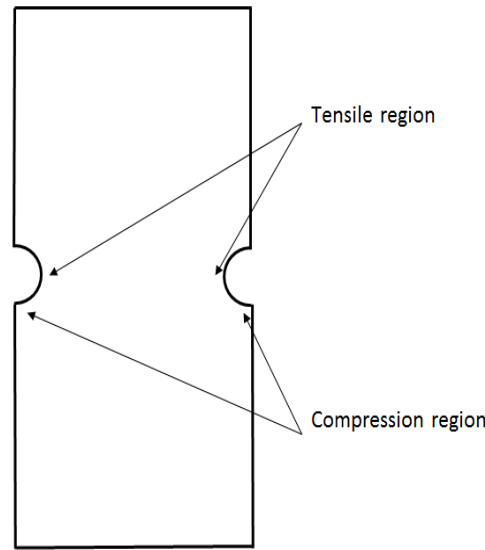

Fig. 2. Schematic of the plate tensile and compression region

\section{Problem formulation}

A plate with central opposite semicircular notch with an integrated piezoelectric patch subjected to a uniaxial external tension is considered as shown in the Fig. 1. Two piezoelectric actuators were placed throughout the plate width. In this work, the stress concentration factors $K_{t}$ is given as $[1,13]$ :

$K_{t}=\frac{\sigma_{\text {max }}}{\sigma_{\text {nom }}}$

where $\sigma_{\max }$ and $\sigma_{\text {nom }}$ are maximum and nominal stress respectively. The piezoelectric actuator placed near to the region of the tensile/compression stress of the host plate and subjected to electric filed. The electric field produces compression/ tension stresses on the patch which will transfer to the host plate over infinitesimal distance [14]. The stress produces by the piezoelectric actuator will change the stress/strain distribution on the region of the tensile and compression stresses of the host plate (Fig. 2).

The electromechanical constitutive equations for the response of the piezoelectric material are [16]:

$\{T\}=\left[c^{E}\right]\{S\}-[e]^{T}\{E\}$,

$\{D\}=[e]\{S\}+\left[\varepsilon^{S}\right]\{E\}$, 
where $\{T\}=\left\{T_{11} T_{22} T_{33} T_{23} T_{13} T_{12}\right\}^{T}$ is the stress vector, $\{D\}=\left\{\begin{array}{lll}D_{1} & D_{2} & D_{3}\end{array}\right\}$ the electric displacement, $\{S\}=\left\{S_{11} S_{22} S_{33} 2 S_{23} 2 S_{13} 2 S_{12}\right\}^{T}$ matrix, [ $\varepsilon$ ] the dielectric constant matrix, [c] the elasticity constant matrix. Superscripts $S$ and $E$ indicate the value at constant $S$ and $E$ respectively.

\section{Finite element analysis}

\subsection{Material and geometrical properties}

The plate and the piezoelectric actuator dimensions are $L=200 \mathrm{~mm}, H=40 \mathrm{~mm}$, $L_{1}=L_{2}=0.5 \mathrm{~L}, r=5 \mathrm{~mm}, h_{P}=h_{a d}=20 \mathrm{~mm}, w_{P}=w_{a d}=0.1 \mathrm{~L} \mathrm{~mm}, t_{P}=0.5 t$, and $t_{a d}=0.03 \mathrm{~mm}$. The material properties are as in shows Table 1 .

\subsection{Finite element model}

Generally, the high gradients of the strain/stress in the region of the discontinuities used to determine the SCFs. Therefore, very fine mesh near the discontinuities is inevitable to capture accurate SCFs. In the present study, the FEM model is carried out using a commercial code Ansys 17.0. Element SOLID186 employed to model the host plate and the adhesive layer while the coupled field element SOLID226 was used for the piezoelectric actuator. Only one- half of the plate with appropriate boundary constraints was modeled due to symmetry.

Table 1. Materials properties of the aluminum, PIC151 and Adhesive

\begin{tabular}{|c|c|c|c|}
\hline Parameter & Aluminum & PI 151 patch & Adhesive \\
\hline Density & $2715 \mathrm{Kg} / \mathrm{m}^{3}$ & $7800 \mathrm{Kg} / \mathrm{m}^{3}$ & $1000 \mathrm{Kg} / \mathrm{m}^{3}$ \\
\hline Poisson ratio & 0.33 & 0.34 & 0.3 \\
\hline Young's modulus & $68.0 \times 10^{9} \mathrm{~N} / \mathrm{m}^{2}$ & & $5.09 \times 10^{9} \mathrm{~N} / \mathrm{m}^{2}$ \\
\hline
\end{tabular}

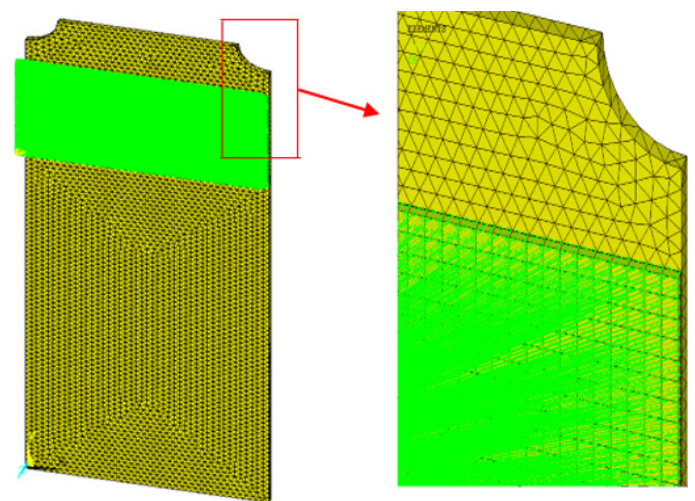

Fig. 3. Finite element model of the host structure and the piezoelectric actuator

\section{Results and discussion}

The nodal stress distributions in area of the semicircular notches without actuator, with passive actuator and active piezoelectric actuator (with negative $100 \mathrm{~V}$ ) are as depicted in the Fig. 4 The applied negative electric filed will cause the patch to subject to positive strain. The figure illustrated that the plate nodal stress decreases with the active piezoelectric actuator. The high stress near the notches was redistributed due to the counteract stresses generated by the piezoelectric actuator around the notch in the way that results in reduction of the SCFs. Another noticed point that the reduction of the stress concentration can be seen after application of external voltage is accompanied with increase in the high tensional stress areas while the compression stress area on the host plate decreases. 


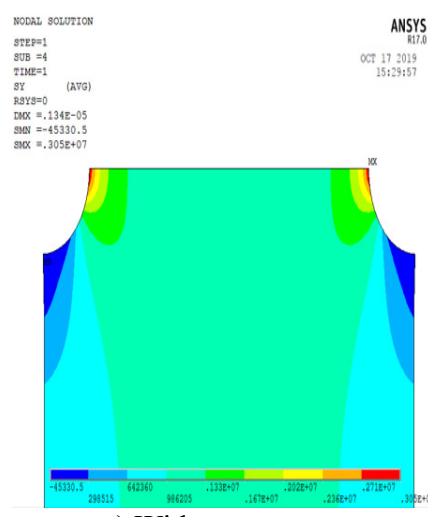

a) Without actuator

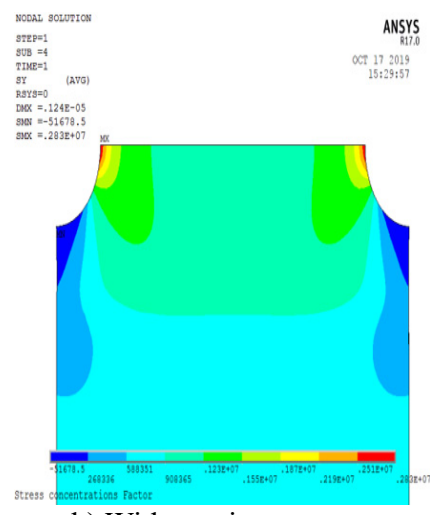

b) With passive actuator

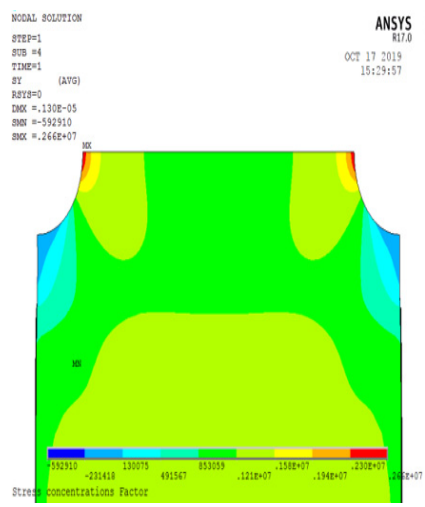

c) With active piezoelectric actuator

Fig. 4. Distributions of the nodal stress

Fig. 5 shows the effect of the positive/negative electric field on the SCFs when the piezoelectric actuators placed in the region of the high tensile stress. The result indicate that the negative electric field redistribute the stress near the notches in such manner that the result SCFs reduced, while the positive electric field leads the SCFs to increase.

The SCFs at the semicircular notches for various negative electric filed with a piezoelectric actuator placed near to compression and tensile zones $\left(h_{P}=H\right)$ and adjacent to the semicircular notch lower edge $\left(L_{e}=r\right)$, illustrated in Fig. 6 . The results indicate that SCFs declines with the increase in negative electric filed due to compression stresses generated by the actuator in the surrounding tensile and compression region of the host plate. It observed that the maximum stress is dropping down with application of the piezoelectric actuators and after that the relation with the applied electric field become linear and proportional to the applied electric field. This drop of the stresses value is attribute to the passive stiffness of the piezoelectric actuators.

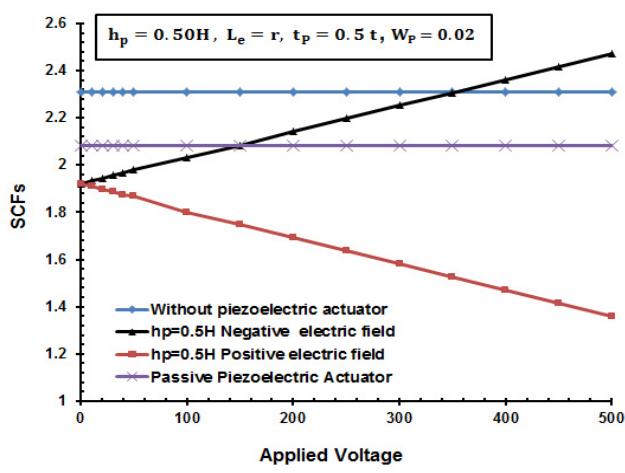

Fig. 5. The effects of positive/negative electric filed on the SCFs

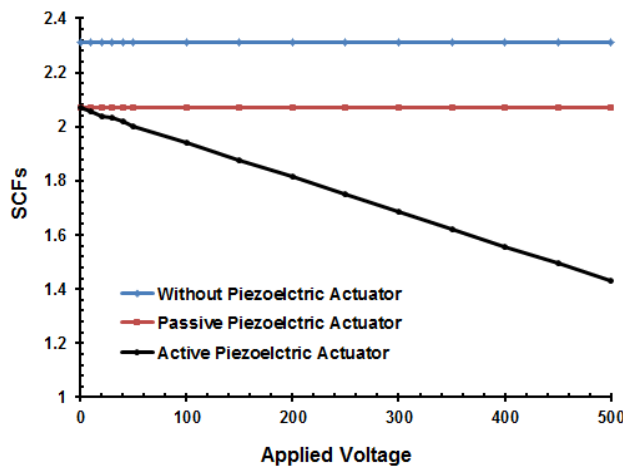

Fig. 6. SCFs variation with negative electric Field

Fig. 7 shows the variation of SCFs with the piezoelectric actuators distance from the notches center line Le. The electric filed is applied such that it produces compression stress on the host plate. The results depict that the reduction of the SCFs with applications of the voltage is conditional, and it will be less efficient when the piezoelectric actuators is located too far from the semicircular notch edges. This is associated to piezoelectric actuator effectiveness distances. As result, placing the piezoelectric actuators far from the notches will give small reduction on SCFs. Consequently, to obtain high reduction of the SCFs its good idea to place the actuator near the semicircular notches. 


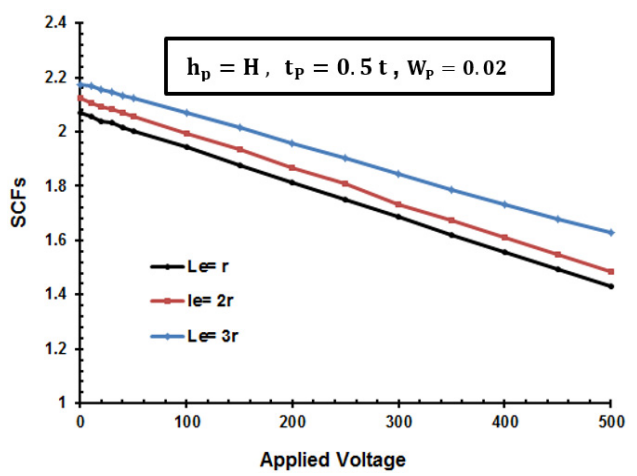

Fig. 7. SCFs variation with piezoelectric actuators distance from the center line $L_{p}$

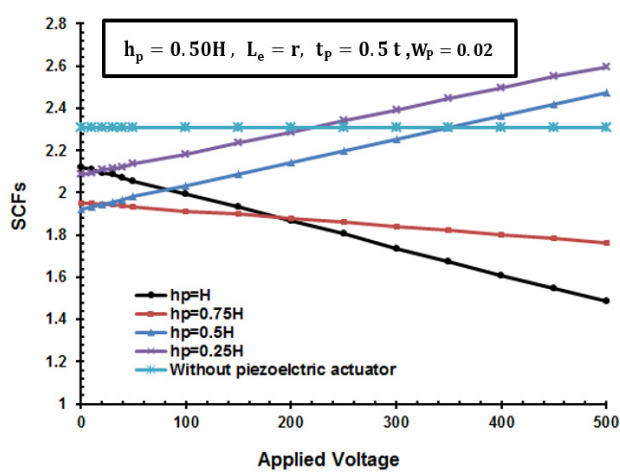

Fig. 8. The variation of SCFs with the piezoelectric actuators length $h_{p}$

The effects of the piezoelectric actuator's length $h_{p}$ on the SCFs with negative electric filed is illustrated in Fig. 8. The figure shows that the when the piezoelectric actuators placement cover any region of the compression zone of the host plate the trend of the reduction is similar and lead to mitigations of the SCFS $\left(h_{p}=H\right.$ and $\left.h_{p}=0.75 H\right)$. Alternatively, in the case that the placement of piezoelectric actuators length cover only the region of the tensile zone of the host plate $\left(h_{p}=0.50 \mathrm{H}\right.$ and $\left.h_{p}=0.25 \mathrm{H}\right)$, the stress concentrations increases with applications of the electric field until it rises beyond the initial stress concentration without piezoelectric actuators. The distributions of the stresses show a superposition of the far stresses field from the tension and the stresses from the piezoelectric actuators which finally lead to increase of the stresses in vicinity of the semicircular notches. However, in order to reduce the SCF, the piezoelectric actuators in the zone of the high tensile stress gradients can be applied with positive electric field to produce compression stress on the patch.

Fig. 9 shows the SCFs variation versus the piezoelectric actuator thickness tp. Increasing the piezoelectric actuators thickness increases the host plat passive stiffness which lead to reduction of the initial SCFs. The relation between the reduction of the SCFs and the piezoelectric actuators thickness is large for relatively low negative voltages (less than $400 \mathrm{~V}$ ).

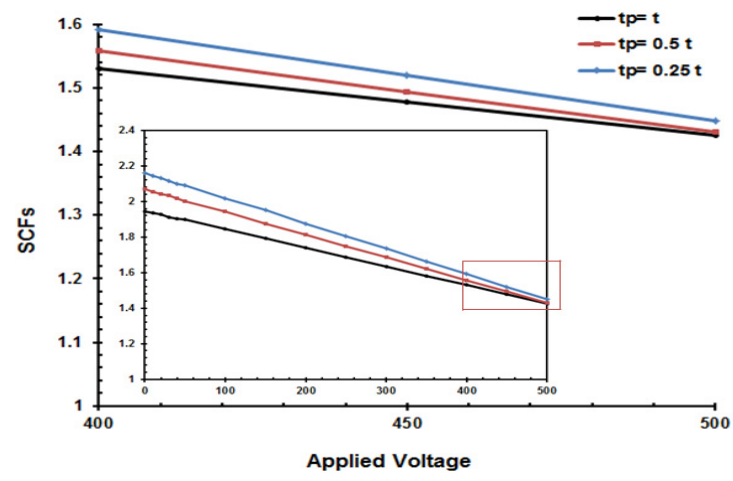

Fig. 9. The variation of SCFs with the piezoelectric actuators thickness $t_{P}$

\section{Conclusions}

The effect of the piezoelectric actuator on the SCFs has been investigated for a rectangular plate with opposite semicircular notches under static uniaxial external loading. The results demonstrate the effectiveness of the piezoelectric actuator in the control and mitigations of SCFs. However, this effectiveness is dependent of the apply electric field, actuator location from the notch and the magnitude of the applied voltage. The results indicate that SCFs reduces linearly 
with active piezoelectric actuator. Further research needs to be conducted to optimize the piezoelectric actuator shape and placement to maximize the reductions of SCFs.

\section{References}

[1] Pilkey W. D., Pilkey D. F. Peterson's Stress Concentration Factors, in Peterson's Stress Concentration Factors. John Wiley and Sons, 2008.

[2] Giare G. S., Shabahang R. The reduction of stress concentration around the hole in an isotropic plate using composite materials. Engineering Fracture Mechanics, Vol. 32, 1989, p. 757-766.

[3] Meguid S. A. Finite element analysis of defence hole systems for the reduction of stress concentration in a uniaxially-loaded plate with two coaxial holes. Engineering Fracture Mechanics, Vol. 25, 1986, p. 403-413.

[4] Wu Z. Optimal hole shape for minimum stress concentration using parameterized geometry models. Structural and Multidisciplinary Optimization, Vol. 37, 2009, p. 625-634.

[5] Liu T. J. C. Fracture mechanics and crack contact analyses of the active repair of multi-layered piezoelectric patches bonded on cracked structures. Theoretical and Applied Fracture Mechanics, Vol. 47, 2007, p. 120-132.

[6] Abuzaid A., Hrairi M., Dawood M. S. Survey of active structural control and repair using piezoelectric patches. Actuators, Vol. 4, 2015, p. 77-98.

[7] Alaimo A., Milazzo A., Orlando C. On the dynamic behavior of piezoelectric active repair by the boundary element method. Journal of Intelligent Material Systems and Structures, Vol. 22, 2011, p. 2137-2146.

[8] Rogers C. A. Intelligent material systems - the dawn of a new materials age. Journal of Intelligent Material Systems and Structures, Vol. 4, 1993, p. 4-12.

[9] Shah D., Chan W., Joshi S. Finite element analysis of plate with piezoelectric layers. 34th Structures, Structural Dynamics and Materials Conference, 1993.

[10] Fesharaki J. J., Golabi S. I. Effect of stiffness ratio of piezoelectric patches and plate on stress concentration reduction in a plate with a hole. Mechanics of Advanced Materials and Structures, Vol. 24, Issue 3, 2017, p. 253-259.

[11] Fesharaki J. J., Madani S. G., Golabi S. I. Best pattern for placement of piezoelectric actuator in classical plate to reduce stress concentration ding PSO algorithm. Mechanics of Advanced Materials and Structures, Vol. 27, Issue 2, 2017, p. 141-151.

[12] Fesharaki J. J., Golabi S. I. A novel method to specify pattern recognition of actuators for stress reduction based on Particle swarm optimization method. Smart Structures and Systems, Vol. 17, Issue 5, 2016, p. 725-742.

[13] Abuzaid A., Hrairi M., Dawood M. S. Estimation of stress concentration factor of plate with hole using piezoelectric actuator and finite element method. IOP Conference Series: Materials Science and Engineering, 2017.

[14] Tada H., Paris P. C., Irwin G. R. The Stress Analysis of Cracks Handbook. 3rd Edition 2000.

[15] Crawley E. F., De Luis J. Use of piezoelectric actuators as elements of intelligent structures. AIAA Journal, Vol. 25, 1987, p. 1373-1385.

[16] ANSI/IEEE Std 176-1987. IEEE Standard on Piezoelectricity, 1988. 